patient-specific variation in the ratios of the infected cell types that might explain why one patient may develop ATLL and another individual develops HAM/TSP? It is predictable that future work will seek to uncover the dynamics of multiple virusinfected cell populations and what impact these forces have on the selection and manifestation of HTLV-1-related diseases.

Address correspondence to: O. John Semmes, Departments of Microbiology and Molecular Cell Biology and Pathology, Eastern Virginia Medical School, Norfolk, Virginia 23501, USA. Phone: (757) 446-5904; Fax: (757) 4465766; E-mail: semmesoj@evms.edu.

1. Hirayama, M., et al. 1988. Infection of human T-lymphotropic virus type I to astrocytes in vitro with induction of the class II major histocompat- ibility complex. Neurosci. Lett. 92:34-39.

2. Hoffman, P.M., et al. 1992. Human T-cell leukemia virus type I infection of monocytes and microglial cells in primary human cultures. Proc. Natl. Acad. Sci.U. S. A. 89:11784-11788.

3. Sibon, D., et al. 2006. HTLV-1 propels untransformed $\mathrm{CD}^{+}$lymphocytes into the cell cycle while protecting $\mathrm{CD}^{+}$cells from death. J. Clin. Invest. 116:974-983. doi:10.1172/JCI27198.

4. Jeang, K.T., Giam, C.Z., Majone, F., and Aboud, M. 2004. Life, death, and tax: role of HTLV-I oncoprotein in genetic instability and cellular transformation. J. Biol. Chem. 279:31991-31994.

5. Marriott, S.J., and Semmes, O.J. 2005. Impact of HTLV-I Tax on cell cycle progression and the cellular DNA damage repair response. Oncogene. 24:5986-5995.

6. Chlichlia, K., et al. 1997. ICE-proteases mediate HTLV-I Tax-induced apoptotic T-cell death. Oncogene. 14:2265-2272.

7. Chlichlia, K., et al. 1995. Immediate effects of reversible HTLV-1 tax function: T-cell activation and apoptosis. Oncogene. 10:269-277.

8. Copeland, K.F., Haaksma, A.G., Goudsmit, J., Krammer, P.H., and Heeney, J.L. 1994. Inhibition of apoptosis in T cells expressing human $\mathrm{T}$ cell leukemia virus type I Tax. AIDS Res. Hum. Retroviruses. 10:1259-1268.

9. Yamada, T., et al. 1994. The human T-cell leukemia virus type I Tax protein induces apoptosis which is blocked by the Bcl-2 protein. J. Virol. 68:3374-3379.

10. Haoudi, A., Daniels, R.C., Wong, E., Kupfer, G., and Semmes, O.J. 2003. Human T-cell leukemia virus-I tax oncoprotein functionally targets a subnuclear complex involved in cellular DNA damageresponse. J. Biol. Chem. 278:37736-37744.

11. Lemoine, F.J., and Marriott, S.J. 2001. Accelerated $\mathrm{G}(1)$ phase progression induced by the human $\mathrm{T}$ cell leukemia virus type I (HTLV-I) Tax oncoprotein. J. Biol. Chem. 276:31851-31857.

12. Liang, M.H., Geisbert, T., Yao, Y., Hinrichs, S.H., and Giam, C.Z. 2002. Human T-lymphotropic virus type 1 oncoprotein tax promotes S-phase entry but blocks mitosis. J. Virol. 76:4022-4033.

13. Park, H.U., Jeong, S.J., Jeong, J.H., Chung, J.H., and Brady, J.N. 2005. Human T-cell leukemia virus type 1 Tax attenuates gamma-irradiation-induced apoptosis through physical interaction with Chk2. Oncogene. 25:438-447.

\title{
Oxidative stress in sepsis: a redox redux
}

\author{
Jay K. Kolls \\ Children's Hospital of Pittsburgh, Pittsburgh, Pennsylvania, USA.
}

\begin{abstract}
Sepsis and sepsis syndrome are leading causes of mortality throughout the world. It is widely held that sepsis represents a dysregulated innate immune response to an offending pathogen. This immune response is often initiated via microbial products signaling through TLRs expressed on host immune cells. There is increasing evidence that this innate response can be dramatically influenced by the cellular redox state, and thus a better understanding of oxidative regulation of innate immunity could lead to new treatments for sepsis. In this issue of the JCI, Thimmulappa et al. show that nuclear factorerythroid 2-related factor 2 (Nrf2), a member of the "cap'n'collar" family of basic region-leucine zipper transcription factors, which has previously been shown to be involved in the transcription of antioxidant gene expression in response to xenobiotic stress, is also a critical regulator of cellular oxidative stress in sepsis (see the related article beginning on page 984).
\end{abstract}

Despite decades of advances in antibiotic treatment, sepsis remains an elusive killer, with over 750,000 cases per year in North America (1) with a $40-50 \%$ mortality rate in adults. Sepsis is mediated by infectious stimuli, and many of the clinical findings of sepsis can be replicated in experimental animal models using specific bacterial

Nonstandard abbreviations used: IKK, inhibitor of $\kappa B$ kinase; IRAK, IL-1 receptor-associated kinase; IRF-3, IFN regulatory factor 3; NAC, $\mathrm{N}$-acetyl cysteine; Nrf2, nuclear factor-erythroid 2-related factor 2; TIR, Toll/IL-1 receptor; TRIF, TIR domain-containing adaptor inducing IFN- $\beta$.

Conflict of interest: The author has declared that no conflict of interest exists.

Citation for this article: J. Clin. Invest. 116:860-863 (2006). doi:10.1172/JCI28111. products such as LPS (2). The last decade of immunological research has revolutionized how scientists understand the initiation of the innate immune response to invading pathogens. For many offending agents, the TLR family of proteins functions as the host sentinel to invading pathogens. This was first demonstrated in Drosophila melanogaster in 1996 (3), where Toll was shown to regulate the production of the antifungal molecule dorsomycin, and later in mammals, when positional cloning revealed Tlr4 to be the Lps gene product (4). An additional 10 human TLRs have been described that recognize other bacterial products as well as fungi and viruses. These receptors signal via their Toll/IL-1 receptor (TIR) domains using 4 adaptor proteins: (a) MyD88; (b)
TIR domain-containing adaptor inducing IFN- $\beta$ (TRIF); (c) MyD88 adaptor-like/ TIR-associated protein (MAL/TIRAP); and (d) TRIF-related adaptor molecule (TRAM) (2). In the case of LPS signaling through TLR4, the MyD88-dependent pathway is critical for NF- $\mathrm{KB}$ activation and the production of TNF- $\alpha$ whereas the MyD88independent, TRIF-dependent pathway is required for type I IFN production. Based on the fact that 10 human TLRs signal via 4 adaptors and 2 predominant kinases to subsequently regulate the expression of hundreds of genes, the innate immune response has been proposed to be shaped like an hourglass (Figure 1). The top of the hourglass is wide, indicating that 10 TLR proteins recognize a variety of potential offending pathogens, then the hourglass narrows to represent a smaller number of highly conserved TLR adaptor proteins and initial kinases, and then it widens again to reflect the increased number of genes that are transcriptionally activated by NF- $\mathrm{KB}$ and other transcription factors (2). This notion is further supported by the fact that this signaling pathway is markedly conserved among mammalian species, and mutations in this pathway in humans that lead to defective TLR signaling are associated with the development of invasive meningococcal (5) or Legionella 


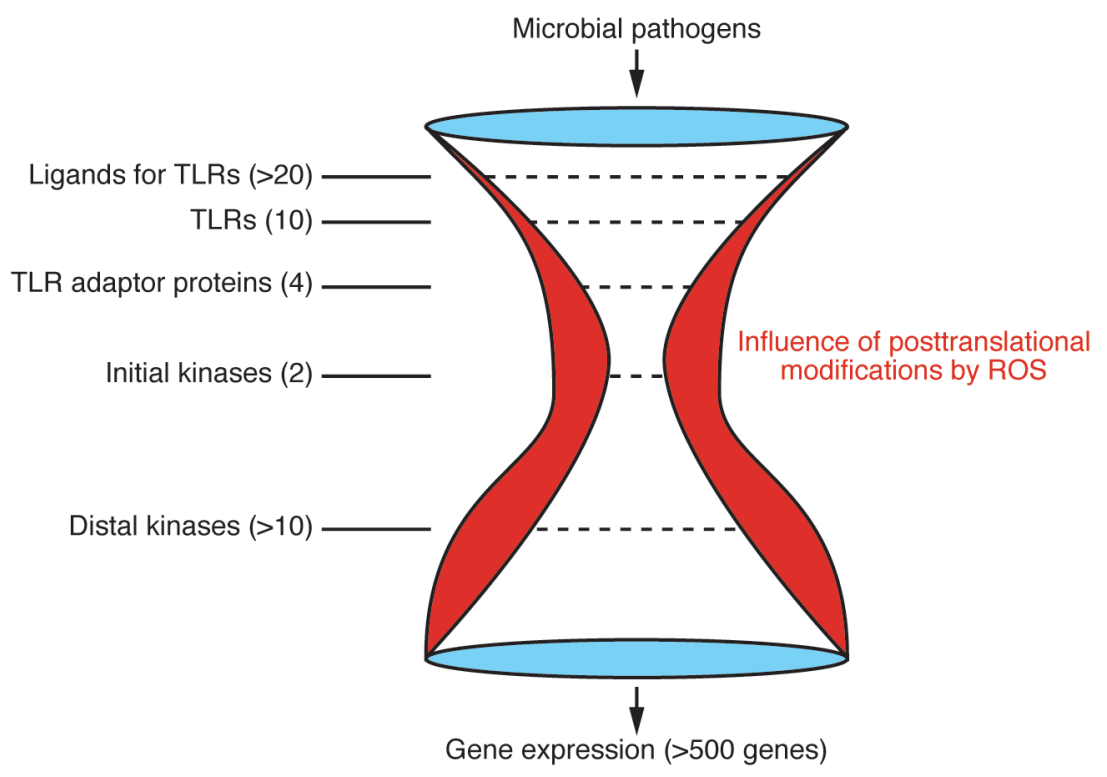

infections (6). The work by Thimmulappa et al. (7) in this issue of the JCI suggests that this hourglass may not be so narrow at its center, as many of the kinases active downstream of TLR signaling can be regulated through oxidant (redox)-dependent posttranslational modifications, resulting in an additional level of control of TLR signaling (Figure 1).

\section{Figure 2}

Potential interactions of Nrf2 and TLR4 signaling. By regulating glutathione S-transferase (GST) and intracellular glutathione (GSH) levels, Nrf2 controls the level of ROS in the cell induced by external stressors such as xenobiotic or electrophilic stress. In this issue of the $\mathrm{JCl}$, Thimmulappa et al. show that the level of ROS regulated by Nrf2 also influences TLR4 signaling at the level of IKK activation, resulting in increased nuclear translocation of NF-KB. In addition, the authors show that IRF-3-mediated gene transcription is also regulated by Nrf2; however, at what level this occurs remains to be determined. Other potential kinase targets of ROS modification include IRAK/IRAK4 and MAPKs as well as TRAF-associated NF- $\mathrm{KB}$ activator-binding kinase 1 (TBK1). ARE, antioxidant response element; CBP, CREB-binding protein; HO-1, heme oxygenase I; Keap1, Kelch-like erythroid cell-derived protein with cap'n'collar homology-associated protein; Maf, mammary cell-activating factor; NQO1, quinone oxidoreductase; TIRAP, TIR-associated protein; TRAM, TRIF-related adaptor molecule.

\section{Nrf2 and oxidative stress}

Redox-dependent control of TLR4 signaling stems from the fact that many of the kinases, transcription factors, and subsequent gene products induced by the TLR4 ligand, LPS, can be posttranslationally modified by ROS (Figure 1). In fact, this has been well studied in the context of $\mathrm{NF}-\kappa \mathrm{B}$ translocation to the nucleus and

\section{Figure 1}

A schematic representation of ROS- and TLR-mediated gene expression. Despite the diversity of microbes that are potential pathogens, there is precise molecular recognition of microbial products by molecules of the innate immune system, with the TLR family being 1 of the most intensely studied mediators of this recognition. There are 10 human TLRs that signal via 4 adaptor proteins and 2 initial kinases; this signaling is followed by the activation of distal kinases that subsequently regulate transcription factors such as NF- $\mathrm{KB}$ and activator protein 1 (AP-1), which control gene expression. Although the signaling cascade is quite narrow at its center, posttranslational modifications of kinase activity by ROS likely contribute to the diversity and intensity of gene expression after microbial activation of the innate immune system. activation of activator protein 1 (AP-1) - 2 transcription factors that regulate gene expression after LPS stimulation of macrophages $(8,9)$. Due to the many sources of ROS within the cell, it has been difficult to take a reductionist approach to identify the critical regulatory factors that control oxidant production at the subcellular level. Moreover, the molecular targets of ROS are

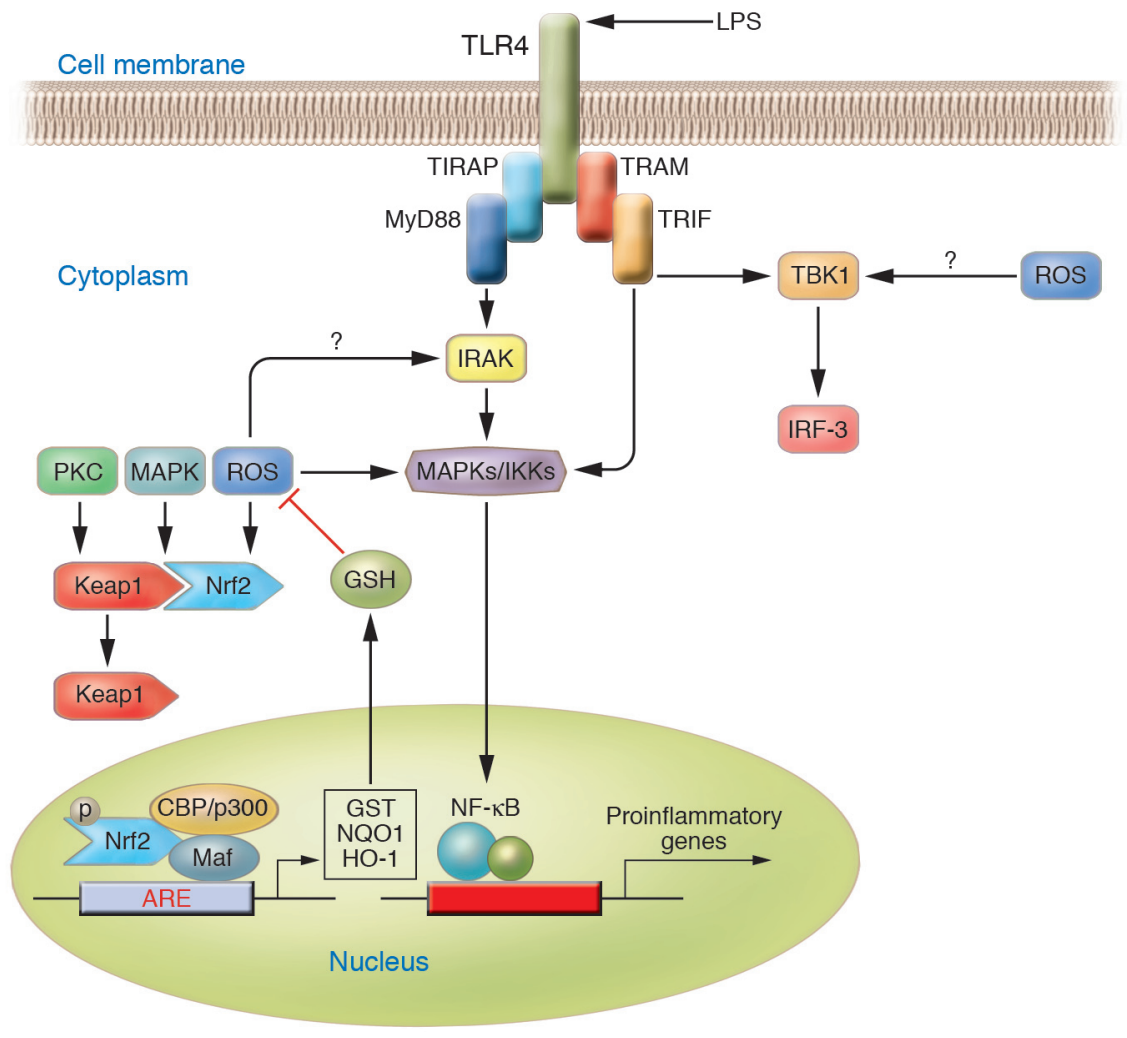


broad and pleiotropic and include proinflammatory events, such as increased production of cytokines, but also activation of antiinflammatory molecules, such as IL-10 and soluble TNF receptors (10). The study by Thimmulappa et al. shows that nuclear factor-erythroid 2-related factor 2 (Nrf2, also known as Nfe2l2), which encodes a basic region-leucine zipper transcription factor, is a key regulator of the cellular redox state in sepsis, in part by regulating levels of intracellular glutathione, a key antioxidant (7). Nrf2 activity is held in check by Kelch-like, erythroid cell-derived protein with cap'n'collar homology-associated protein 1 (Keap1), but upon cellular activation, such as oxidative stress or MAPK activation, it dissociates and translocates to the nucleus where it binds to cis-acting antioxidant response elements, which regulate the expression of antioxidant and phase II detoxification genes (11) (Figure $2)$. Studies of mice with a homozygous deletion of $\mathrm{Nrf} 2$ show that $\mathrm{Nrf} 2$ is critical for the hepatic induction of glutathione S-transferase (GST) and NAD(P)H:quinone oxidoreductase (NQO1) in response to phenolic antioxidants (12). Moreover, these mice develop hemolytic anemia, presumably due to increased lipid peroxidation (13). Although Nrf2 is clearly critical for regulating the antioxidant response and phase II detoxification (Figure 2) of certain xenobiotics, such as acetaminophen (14), its role in innate immunity had not been previously investigated.

\section{Nrf2, ROS, and kinase activation}

In the current study, mice deficient in Nrf2 displayed increased mortality in both a sterile (LPS administration) and a nonsterile (cecal ligation and puncture) model of sepsis (7). Moreover, Nrf2 $2^{-1-}$ mice had increased expression levels of TNF and lung injury after systemic LPS administration. Using gene expression profiling, Thimmulappa et al. demonstrated that $N r f 2$ regulates a number of key proinflammatory cytokines, including IL-23p19, IL-1F9, and IL-6, as well as chemokines, including CCL8, CCL6, CCL9, CCL2 (also known as MCP-1), and CXCL10. These mice also demonstrated increased expression of genes coding for proinflammatory molecules, such as TNF, IL-1 $\beta$, and IL-6, after stimulation with TNF in vivo. However, it remains unclear what proportion of this dysregulated gene expression is a direct effect of $\mathrm{Nrf2}$ deficiency versus a downstream effect of the dysregulation of, for example, TNF.
However, some of the data suggest that Nrf2 may be acting at the level of kinase activation downstream from TLR4 (Figure 2). Fibroblasts from $\mathrm{Nrf2^{-/- }}$ mice showed higher levels of inhibitor of $\kappa \mathrm{B}$ kinase (IKK) activity, increased IкB- $\alpha$ phosphorylation, and more rapid nuclear translocation of NF-кB (7). Moreover, the effect of Nrf2 was not just relegated to the MyD88-dependent (Figure 2) pathway; Nrf2 also had effects on the MyD88-independent, TRIF-dependent pathway as evidenced by the upregulation of IFN regulatory factor 3-dependent (IRF-3-dependent) genes as well as transactivation of an IRF-3-dependent reporter gene in vitro in $\mathrm{Nrf2^{-/- }}$ fibroblasts. Based on these data, it would be important to determine whether Nrf2 is regulating the upstream kinases IL-1 receptor-associated kinase (IRAK) and IRAK4, which are critical for MyD88-dependent signaling, or TNF receptor-associated factor-associated NF- $\mathrm{KB}$ activator-binding kinase 1 (TBK1) (Figure 2) (15), which is critical for IRF-3mediated transcription, or if Nrf2 is asserting its regulatory effects downstream from these kinases at the level of the MAP kinases, in addition to its described effects on distal kinases, such as IKK (Figure 2). Since IL-23p19 is upregulated in $\mathrm{Nrf2}^{-/-}$mice, it will also be important to determine if Nrf2 also regulates adaptive Th1 and ThIL-17 responses $(16,17)$, as has been reported for Th2 immune responses (18).

\section{Antioxidants and sepsis}

In support of a role for $\mathrm{Nrf} 2$ as a critical regulator of antioxidant gene expression, restoration of intracellular glutathione with $\mathrm{N}$-acetyl cytseine (NAC) decreased LPS- and TNF-induced NF- $\kappa$ B activation and reduced LPS-induced lung injury in Nrf2 $2^{-1-}$ mice (7). These data confirm the critical role of the cellular redox state in regulating innate immune responses and support the contention that the transcriptional regulation of the antioxidant response is critical in regulating the cellular response to external stressors. Thus, polymorphisms may exist in the Nrf2 gene that may identify subjects at risk for more severe sepsis. In a recent clinical trial, NAC was shown to reduce NF- $\mathrm{KB}$ activation as well as IL-8 secretion in patients with sepsis (19). However, in a subsequent randomized trial of 34 patients, NAC failed to improve end-organ function or microalbuminuria (20). This underlying reason for the failure of antioxidant therapy may be similar to that observed for the failure of other anti- inflammatory approaches: the timing of these interventions may be too late to adequately interfere with the induction of the inflammatory cascade. Thus, early administration of antioxidant-based therapy is likely critical. A potential advantage of an antioxidant approach is that restoration of normal cellular glutathione levels should leave basal innate immunity intact. However, it remains unclear which subcellular stores of glutathione need to be restored. Guidot et al. have shown that NAC preferentially repletes cytosolic glutathione stores, but not mitochondrial stores (21). Therefore, we need to better understand redox regulation of TLR signaling at the subcellular level in order to propose a rational redox-based therapy for a disease as complex as sepsis.

\section{Acknowledgments}

The author would like to thank Bruce Beutler for his critical review of the manuscript.

Address correspondence to: Jay K. Kolls, Children's Hospital of Pittsburgh, Suite 3765, 3705 Fifth Avenue, Pittsburgh, Pennsylvania 15213, USA. Phone: (412) 648-7457; Fax: (412) 692-6645; E-mail: jay.kolls@chp.edu.

1. Angus, D.C., et al. 2001. Epidemiology of severe sepsis in the United States: analysis of incidence, outcome, and associated costs of care. Crit. Care Med. 29:1303-1310.

2. Beutler, B. 2004. Inferences, questions and possibilities in Toll-like receptor signalling. Nature. 430:257-263.

3. Lemaitre, B., Nicolas, E., Michaut, L., Reichhart, J.M., and Hoffmann, J.A. 1996. The dorsoventral regulatory gene cassette spatzle/Toll/cactus controls the potent antifungal response in Drosophila adults. Cell. 86:973-983.

4. Poltorak, A., et al. 1998. Defective LPS signaling in $\mathrm{C} 3 \mathrm{H} / \mathrm{HeJ}$ and $\mathrm{C} 57 \mathrm{BL} / 10 \mathrm{ScCr}$ mice: mutations in Tlr4 gene. Science. 282:2085-2088.

5. Smirnova, I., et al. 2003. Assay of locus-specific genetic load implicates rare Toll-like receptor 4 mutations in meningococcal susceptibility. Proc. Natl. Acad. Sci. U. S. A. 100:6075-6080.

6. Hawn, T.R., et al. 2003. A common dominant TLR5 stop codon polymorphism abolishes flagellin signaling and is associated with susceptibility to legionnaires' disease. J. Exp. Med. 198:1563-1572.

7. Thimmulappa, R.K., et al. 2006. Nrf2 is a critical regulator of the innate immune response and survival during experimental sepsis. J. Clin. Invest. 116:984-995. doi:10.1172/JCI25790.

8. Covert, M.W., Leung, T.H., Gaston, J.E., and Baltimore, D. 2005. Achieving stability of lipopolysaccharide-induced NF-kappaB activation. Science. 309:1854-1857.

9. Tanaka, C., Kamata, H., Takeshita, H., Yagisawa, H., and Hirata, H. 1997. Redox regulation of lipopolysaccharide (LPS)-induced interleukin-8 (IL-8) gene expression mediated by NF kappa B and AP-1 in human astrocytoma U373 cells. Biochem. Biophys. Res. Commun. 232:568-573.

10. Zhang, Z., et al. 2001. Reactive oxygen species 
mediate tumor necrosis factor alpha-converting, enzyme-dependent ectodomain shedding induced by phorbol myristate acetate. FASEB J. 15:303-305.

11. Cao, T.T., et al. 2005. Increased nuclear factorerythroid 2 p 45-related factor 2 activity protects SH-SY5Y cells against oxidative damage. J. Neurochem 95:406-417.

12. Itoh, K., et al. 1997. An Nrf2/small Maf heterodimer mediates the induction of phase II detoxifying enzyme genes through antioxidant response elements. Biochem. Biophys. Res. Commun. 236:313-322.

13. Lee, J.M., Chan, K., Kan, Y.W., and Johnson, J.A. 2004. Targeted disruption of Nrf2 causes regenerative immune-mediated hemolytic anemia. Proc. Natl. Acad. Sci. U. S. A. 101:9751-9756.
14. Chan, K., Han, X.D., and Kan, Y.W. 2001. An important function of $\mathrm{Nrf} 2$ in combating oxidative stress: detoxification of acetaminophen. Proc. Natl. Acad. Sci. U. S. A. 98:4611-4616.

15. McWhirter, S.M., et al. 2004. IFN-regulatory factor 3 -dependent gene expression is defective in Tbk1deficient mouse embryonic fibroblasts. Proc. Natl. Acad. Sci. U. S. A. 101:233-238.

16. Kolls, J.K., and Linden, A. 2004. Interleukin-17 family members and inflammation. Immunity. 21:467-476.

17. Langrish, C.L., et al. 2004. IL-12 and IL-23: master regulators of innate and adaptive immunity. Immunol. Rev. 202:96-105.

18. Rangasamy, T., et al. 2005. Disruption of Nrf2 enhances susceptibility to severe airway inflamma- tion and asthma in mice. J. Exp. Med. 202:47-59.

19. Paterson, R.L., Galley, H.F., and Webster, N.R. 2003. The effect of $\mathrm{N}$-acetylcysteine on nuclear factorkappa B activation, interleukin-6, interleukin-8, and intercellular adhesion molecule- 1 expression in patients with sepsis. Crit. Care Med. 31:2574-2578.

20. Spapen, H.D., Diltoer, M.W., Nguyen, D.N., Hendrickx, I., and Huyghens, L.P. 2005. Effects of Nacetylcysteine on microalbuminuria and organ failure in acute severe sepsis: results of a pilot study. Chest. 127:1413-1419.

21. Guidot, D.M., and Brown, L.A. 2000. Mitochondrial glutathione replacement restores surfactant synthesis and secretion in alveolar epithelial cells of ethanol-fed rats. Alcohol. Clin. Exp. Res. 24:1070-1076.

\title{
The alchemy of tendon repair: a primer for the (S)mad scientist
}

\author{
Dwight A. Towler ${ }^{1}$ and Richard H. Gelberman ${ }^{2}$
}

\author{
1Department of Medicine, Division of Bone and Mineral Diseases and \\ ${ }^{2}$ Department of Orthopedic Surgery, Washington University School of Medicine, St. Louis, Missouri, USA.
}

\begin{abstract}
During vertebrate development, mesenchymal progenitors capable of forming bone, cartilage, muscle, fat, or tendon arise from either neural crest or somitic mesoderm. Transcriptional programs that specify mesenchymal cell fates are initiated and modified by paracrine cues provided by TGF- $\beta$ superfamily members and mediated in part via the regulated assembly of Smadcontaining multiprotein transcription factor complexes. In this issue of the JCI, Hoffmann and colleagues have identified that Smad8 activation drives tendon formation from $\mathrm{C} 3 \mathrm{H} 10 \mathrm{~T} 1 / 2$ cells, a murine cell line that recapitulates many features of normal multipotent mesenchymal cells (see the related article beginning on page 940). Cells programmed to the tenocyte cell fate in vitro formed tenogenic grafts in vivo. These results add to the accumulating evidence that proliferating, multipotent mesenchymal progenitor cells can be programmed to yield multiple cell types - e.g., osteoblasts, myocytes, chondrocytes, and tenocytes - that may be useful in cell-based therapeutic approaches to musculoskeletal diseases.
\end{abstract}

Tendon and ligament injuries represent some of the most common musculoskeletal disorders that clinicians address daily, ranging from as mundane as a mild ankle sprain to the crippling effects of Achilles tendon rupture or flexor tendon injury in the hand (1). Severe tendon injuries are difficult to manage. Surgical repairs frequently do not fully restore function due to fibrous adhesions or failure arising from the mechanical demands placed on

Nonstandard abbreviations used: BMP, bone morphogenetic protein; GDF, growth differentiation factor; L+MH2, R-Smad linker plus MH2 domain; R-Smad, receptor-regulated Smad.

Conflict of interest: The authors have declared that no conflict of interest exists.

Citation for this article: J. Clin. Invest. 116:863-866 (2006). doi:10.1172/JCI28320. imperfect integrative healing at tendontendon or tendon-bone interfaces (1).

The tendon itself is a dense, regular connective tissue consisting primarily of type I collagen and the interspersed specialized mesenchymal cells known as tenocytes that are responsible for the maintenance of collagen structure, with avascular bundles of primary fibers invested by a thin layer of endotenon, a fine loose connective tissue sheath, to form fascicles (2) (Figure 1). Parallel arrays of fascicles are bundled together to form tendon by the epitenon, a layer contiguous with the endotenon through which the microvasculature, lymphatics, and innervation delicately traverses (2).

The ontogeny of the tenocyte lineage is only beginning to be understood. Elegant work recently forthcoming from the Tabin laboratory has demonstrated that during embryogenesis, a unique compartment of the somite called the syndetome provides tenocyte progenitors (3). Inductive interactions between the well-described myotome and sclerotome layers generate the syndetome, demarcated at the earliest stages of development by expression of the basic helix-loop-helix transcription factor gene Scleraxis. In addition to promoting tenocyte "birth," the myoprogenitors of the myotome appear to prevent chondrogenic differentiation of tenocyte progenitors. The paracrine signals that fine-tune multipotent mesenchymal progenitors to the unique tenocyte fate during normal development are virtually unknown, but appear to involve FGFs (3) and key members of the TGF- $\beta$ superfamily such as growth differentiation factor 5 (GDF5) and GDF7 (4).

\section{A tenogenic Smad}

Thus, given our limited understanding of how mesenchymal progenitors are efficiently recruited to the tenocyte lineage, the recent progress made by Hoffmann and colleagues in a report in this issue of the JCI is quite remarkable (5). Their insights into the mechanisms controlling tenocyte differentiation arose from fortuitous observations made while studying Smad signaling in C3H10T1/2 cells - a murine multipotent mesenchymal cell line that recapitulates many features of par- 